\title{
La aplicación del programa europeo de desarrollo rural LEADER + en España y la comunidad valenciana
}

\author{
The Application of the European Rural Development \\ Program LEADER + in Espain \\ and the Valencian Community
}

\author{
Jordi Tormo i Santonja \\ Grupo Interdisciplinario de Estudios \\ Críticos y de América Latina (GIECRYAL) \\ Departamento de Geografía Humana. \\ Universidad de Alicante (España) \\ Facultad de Filosofía y Letras. \\ Campus de San Vicente del Raspeig
}

\section{Resumen}

Las políticas de desarrollo rural en la Unión Europeo abogaron por la promoción de la multifuncionalidad de la agricultura hasta la reforma de la Política Agraria Común de 1992. Desde este momento, las políticas europeas de desarrollo rural pasaron a propugnar un nuevo modelo de desarrollo sustentado en la multifuncionalidad del medio rural y no de la agricultura.

Los programas LEADER son un claro ejemplo de la aplicación de las nuevas iniciativas europeas para la promoción de la multifuncionalidad del medio rural, ya que las líneas de ayuda de estos programas han prestado una especial atención al turismo rural, la valorización de los recursos naturales, culturales y arquitectónicos con fines turísticos y la dotación de servicios a la población.

Palabras clave: Desarrollo Rural, Europa, España, Comunidad Valenciana, GAL CEDER-Aitana, LEADER. 


\begin{abstract}
Abstrac
Until the Common Agricultural Policy was reformed in 1992, rural development policies in the European Union advocated a multifunctionality approach to agriculture. After that point, EU policies supported a new model of rural development, based on the multifunctionality of the rural environment, rather than of agriculture.

The LEADER programmes are a clear example of how these new EU initiatives have been implemented to stimulate multifunctionality in rural environments, as the criteria for aid contemplated within these programmes is geared towards consolidating rural tourism, the valuation of natural, cultural and architectural resources for tourist purposes and providing services to the local populations.
\end{abstract}

Keywords: Rural development, Europe, Spain, Region of Valencia, GAL CEDER-Aitana, LEADER.

\title{
1. De la multifuncionalidad agraria a la multifuncionalidad rural: el impacto de la política agraria común en el medio rural europeo
}

La agricultura en la Unión Europea está regulada por la Política Agraria Común (PAC), cuyo objeto inicial fue la mejora del equilibrio territorial y de la cohesión socioeconómica mediante el fomento de la actividad agraria. El Tratado de Roma (1958) fijó los fundamentos de la PAC. Su finalidad principal era asegurar el abastecimiento alimenticio a los países europeos, tras la situación de carestía de alimentos que había sufrido Europa durante los años cincuenta. Para ello se establecieron los fondos y ayudas europeas de FEOGA-Orientación y FEOGA-Garantía a partir de 1962, con los que se conseguían mantener los precios de las producciones agrarias.

La PAC es una estrategia de intervención pública a nivel europeo que tuvo como función, en su origen, promover la consolidación del desarrollo 
social y económico de las áreas rurales y garantizar el autoabastecimiento de los productos agrarios de acuerdo a los principios de la unidad del mercado europeo, la preferencia europea de la producción, la solidaridad financiera entre los Estados miembro y la no discriminación. Según J. A. Segrelles (2008, p. 11), la PAC contenía implícitos los siguientes preceptos básicos:

- "La homogeneidad de la función social del medio rural.

- La pertinencia de un paradigma centrado en la modernización del sistema productivo.

- La presunción de que se podrían superar todos los problemas rurales con una única herramienta política.

- La explicitación del modelo de agricultura familiar como objeto prioritario de la política comunitaria.

- La conveniencia de integrar los objetivos sectoriales y sociales en una misma política".

Sin embargo, según J. A. Segrelles (2007) y J. D. Gómez (2008), las presiones internacionales, la creciente generación de excedentes y el elevado gasto presupuestario de las políticas agrícolas han modificado su inicial modelo de desarrollo "agrarista" por un modelo de carácter "ruralista" que se apoya en una serie de estrategias como son la multifuncionalidad del medio, la pluriactividad de las rentas, el desarrollo rural sostenible y la calidad de los alimentos, entre otros. Ahora, tras la reforma de 1992, el objeto de la PAC es mantener la eficiencia productiva y la competitividad de la agricultura europea en los mercados internacionales mediante la reducción de las producciones, los excedentes y los gastos presupuestarios. En concreto, según J. Romero y J. Farinós (2004), este cambio en la política agraria se sustenta en el establecimiento de incentivos económicos para la reducción de la producción de alimentos a fin de disminuir los excedentes generados por las explotaciones agrícolas industrializadas y sostener las áreas rurales tradicionales, en las que la función social y económica de la agricultura es cada vez menor. 
La reforma de la PAC se inició con la aprobación en enero de 1991 del documento de trabajo Evolución y futuro de la PAC, también conocido como Informe Mac Sharry. Según J. J. Romero (2002, p. 135), se establecían los siguientes objetivos en la reforma de la PAC:

- "Mantener la población rural y ligar la función productiva del agricultor con la protección del medio ambiente.

- Ligar la función productiva agraria con la protección del medio ambiente.

- Diversificación de las actividades del campo.

- Controlar los excedentes y el gasto.

- Mantener la política de precios con otras fórmulas. Sustituir las ayudas a precios por ayudas directas a los agricultores.

- Dirigir los sistemas de apoyo a compatibilizar el equilibrio de los mercados, con una producción de calidad, respetuosa con el medio ambiente.

- Asumir la creciente interdependencia internacional y las responsabilidades de ser el principal importador mundial de alimentos, y el segundo exportador.

- Reafirmar los principios tradicionales de la PAC: unidad de mercado, preferencia comunitaria y solidaridad financiera.

- Convertir el presupuesto agrario en un instrumento real de solidaridad financiera, a favor de los más necesitados.

- Incrementar las medidas de estabilización ya existentes, como las cuotas nacionales o el abandono de tierras, proporcionalmente al tamaño de las explotaciones".

La reforma de la PAC ha tenido entre sus principales cambios de enfoque el control y la limitación de las producciones a través del establecimiento de cuotas, el fomento de la pluriactividad rural y el cambio de enfoque de las ayudas que pasaron de sostener los precios a ser directas para los agricultores. En concreto, en noviembre de 1996 los países miembro de la Unión Europea aprobaron la Declaración de Cork. Se trata de un acuerdo en el que se establecía la necesidad de cambiar 
los vigentes pagos compensatorios a los agricultores por unas nuevas líneas de ayudas directas. Las pautas básicas de la Declaración de Cork establecían que las nuevas políticas europeas debían sustentarse en la diversificación, la simplificación, la sostenibilidad y el enfoque integrado de las políticas de desarrollo rural.

Según A. García (2007, p. 68) la reforma de la PAC “concentra las ayudas en pocas manos, que no son, precisamente, las de los pequeños y medianos agricultores/as. Para poder compatibilizar estas políticas con la Organización Mundial del Comercio (OMC) han decidido que puedan cobrarse sin producir, medida que no tiene ni pies ni cabeza y que deslegitima tanto a estas ayudas como a la propia PAC. La última reforma de la PAC es una reforma destructora del modelo de agricultura social". Atrás queda el modelo intensivo y especializado que se fundamentó en la aplicación de la PAC desde finales de los años cincuenta.

A diferencia del primer periodo de aplicación de la PAC, el medio rural ha perdido un importante peso social y político y ha dejado de ser una fuente de votos para los partidos en el gobierno, sobre todo a partir de la urbanización de los países europeos durante los años setenta y ochenta, principalmente. Esta es una de las principales causas del cambio de enfoque de la PAC de 1992. Esta situación ha supuesto que, según J. Pliego (2002, p. 220-221), el medio rural se caracterice por la existencia de "estructuras agrarias deficientes, escasas oportunidades de empleo, infraestructuras y equipamientos inadecuados, débil diversificación del tejido económico, desvalorización social de la actividad agraria y abandono del campo por parte de los sectores más jóvenes y dinámicos".

Estos cambios han motivado la aparición de un nuevo enfoque en las políticas europeas de desarrollo rural, en las que el papel de la agricultura como motor de progreso ha pasado a un segundo lugar. Un buen ejemplo es la iniciativa comunitaria LEADER. El establecimiento de las nuevas políticas de desarrollo se basan en la puesta en valor de las áreas rurales y de interior a partir del desarrollo del turismo y de los servicios, principalmente, y olvidan que la agricultura ha sido la principal fuente de riqueza de este tipo de áreas, que mantiene un paisaje y una cultura ahora explotada desde otra perspectiva, que es lo único que 
vincula a la población con el territorio y que sin agricultura no habrá población, paisaje, cultura ni valores. El desarrollo rural debe basarse en el desarrollo de la agricultura. Sin agricultura no habrá mundo rural ni desarrollo rural. Este medio acabará transformándose en un espacio periurbano, urbanizado y de uso y disfrute de la población urbana.

En síntesis, según R. Crecente (2002), se ha producido una distorsión en la definición del concepto de multifuncionalidad y se ha pasado de basar el desarrollo rural en la multifuncionalidad de la agricultura (producción de alimentos y defensa de los valores paisajísticos, entre otros) a sustentarlo en la multifuncionalidad del espacio agrario (turismo rural e introducción del proceso urbanizador, entre otros).

\section{El programa LEADER: una herramienta para la aplicación de las políticas rurales europeas}

El programa Liasions Entre Activités de Development de l'Economie Rural (Relaciones entre las Actividades de Desarrollo de la Economía Rural, LEADER) es una iniciativa desarrollada por la Unión Europea y los países miembro en materia de desarrollo rural. Este programa europeo está orientado a aquellos territorios susceptibles de llevar a cabo acciones de desarrollo, cuya situación social y económica ponga en peligro su supervivencia, es decir, regiones rurales, en su mayoría de montaña, que necesitan toda una serie de actuaciones con la finalidad de fomentar el desarrollo social, cultural y económico.

Es necesario recordar que el Dictamen del Comité Económico y Social de la Unión Europea "El futuro de las zonas de montaña en la Unión Europea" (2003/C61/19) prestaba especial atención a este tipo de zonas y determinaba que su especificidad era la consecuencia de una serie de factores sobre los que se debía hacer hincapié de forma directa. Entre ellos destacaba la situación demográfica, los servicios públicos, las comunicaciones, el turismo, la utilización de las tierras, el patrimonio natural, los suelos, el agua, la energía, el hábitat, los riesgos naturales y la economía, si bien el papel de la agricultura en el desarrollo rural quedaba relegado a un segundo lugar. 
Una de las principales deficiencias de las políticas europeas de desarrollo rural, y principalmente del programa LEADER, es que consideran al medio rural europeo bajo unos parámetros comunes que quedan establecidos en las convocatorias públicas realizadas por la Unión Europea. Se trata de políticas que no contemplan los diferentes grados de ruralidad de las áreas europeas y su consecuente caracterización en unidades territoriales diferenciadas. Por ello, su implementación es incorrecta, ya que aplica una serie de acciones homogeneizadas a un grupo de áreas rurales diferenciadas entre sí. En consecuencia, la aplicación de estas homogenizadas políticas europeas incrementa las diferencias entre las áreas rurales, ya que no surgen de los condicionantes particulares, sino de los globales.

Las iniciativas de desarrollo que se plantean en las áreas de acción a través de LEADER necesitan de la implicación e interacción de tres agentes clave para el proceso de desarrollo local, los cuales agrupan las diferentes tendencias e iniciativas del territorio como son la población local, los organismos públicos y los organismos privados. Estos agentes deben trabajar conjuntamente para lograr un desarrollo que debe de partir de la escala local integrándose en las estructuras globales, es decir, se deben aprovechar los recursos endógenos y la estructura tradicional para innovar desde la escala local y conseguir, mediante todas las iniciativas europeas, cambios en el medio rural en términos globales. Ahora bien, como se ha indicado arriba, en la escala local se aplica un modelo de actuación homogeneizado desde la Unión Europea, lo que constituye una gran contradicción.

El espacio europeo LEADER se estructura a partir de los Grupos de Acción Local (GAL). Cada GAL aglutina a un número de municipios que básicamente se caracterizan por ofrecer condicionantes y estructuras comunes, así como por su pertenencia a un mismo espacio geográfico, siendo los grupos de acción los encargados de llevar a cabo y apoyar las iniciativas de desarrollo en cada área LEADER de Europa.

El Programa LEADER nació en 1991 en el seno de la Unión Europea y desde entonces se han implantado tres concesiones y etapas de trabajo. Cada etapa ha tenido unas líneas de trabajo diferenciadas. Sin embargo, su objetivo ha sido claro: desarrollar las áreas rurales europeas propiciando 
la multifuncionalidad rural a partir del desarrollo de la economía, la pluriactividad de las rentas y la mejora de la calidad de vida.

La primera etapa de aplicación se denominó LEADER I y se desarrolló entre 1991 y 1993, siendo 52 los GAL beneficiarios de esta política europea en el territorio español. En este primer periodo, la principal apuesta fue el fomento y desarrollo de las iniciativas de turismo rural, ya que equivocadamente se consideró a este sector como un revitalizador de los espacios rurales.

La segunda fase, LEADER II, se desarrolló entre 1994 y 1999 y se beneficiaron de ella 132 GAL en el territorio español. Durante este periodo, las principales líneas de trabajo se basaron en el turismo rural y en la conservación del ambiente, así como en la mejora de las infraestructuras en el medio rural, es decir, servicios sociales, culturales y de comunicación.

La tercera etapa, es decir LEADER +, se implementó entre 2000 y 2006, siendo 145 los GAL beneficiados en el territorio español. Durante la aplicación del programa LEADER + se profundizó en el fomento de los servicios a la población, el turismo rural y en la puesta en valor del patrimonio cultural, natural y de los productos agrarios. Además, se impulsó el establecimiento de redes de trabajo y proyectos internacionales a través de los que fomentar el intercambio de experiencias.

La convocatoria de las ayudas establecidas a través de LEADER ha sido utilizada por la población local y los agentes públicos y privados para la ejecución de acciones en materia de desarrollo de turismo rural como son la puesta en valor de los recursos patrimoniales y de dotaciones y alojamientos rurales. Se ha sobrevalorado la posibilidad real de desarrollo que el turismo de interior puede tener en el medio rural. Un ejemplo de ello es que actualmente la mayor parte de los alojamientos rurales funcionan de forma estacional y registran un bajo índice de ocupación anual.

El desarrollo de la oferta de alojamiento de turismo rural en España, y la puesta de moda de este tipo de prácticas, ha tenido entre sus consecuencias que muchos de los políticos municipales vislumbraran en su desarrollo turístico su futuro económico. Sin embargo, no ha sido 
así. No se debe entender el turismo rural como la única salida a la crisis económica de las áreas rurales, sino como una de las muchas iniciativas que pueden suponer un aporte al desarrollo integral del medio rural. Según H. Capellà (2002, p. 79), el turismo rural ha sido un fracaso en la mayoría de los casos. En su opinión, "los prejuicios externos sobre el mundo rural han sido los verdaderos responsables de una marginación continuada que se ha materializado esta vez en el espejismo del turismo, olvidando las potencialidades y riquezas aún existentes“.

\section{El programa LEADER + (2000-2006). aplicación en Europa y España}

\subsection{Los objetivos y la articulación de LEADER +}

Según la Red Española de Desarrollo Rural (REDR) del Ministerio de Agricultura, Pesca y Alimentación (2008), el objetivo general del programa LEADER + ha sido incentivar y ayudar a los agentes del mundo rural para que reflexionaran sobre el potencial de su territorio en una perspectiva a largo plazo. Además, los objetivos adicionales de LEADER + han sido los siguientes:

- Experimentar nuevas formas de valorización del patrimonio natural y cultural.

- Mejorar el entorno económico con el fin de contribuir a la creación de empleo.

- Mejorar la capacidad de organización de las respectivas comunidades rurales.

Para ello, la Comunicación de la Comisión a los Estados Miembros de la Unión Europea 2000/C 139/05 de 14 de abril de 2000 indicaba que las principales aportaciones de la iniciativa LEADER + a este proceso eran las siguientes:

- Movilizar a los agentes locales para que procedieran a una reflexión y se responsabilizasen del futuro de su territorio. 
- Aplicar un enfoque territorial descentralizado e integrado basado en un proceso ascendente (bottom up).

- Abrir las zonas rurales a otros territorios mediante el intercambio de experiencias y la constitución de redes.

- Posibilitar la financiación de las operaciones de pequeña cuantía.

La Comunicación de la Comisión Europea (2000) fijaba las orientaciones básicas sobre la iniciativa LEADER + y, entre ellas, su articulación. La iniciativa de la Comisión Europea ha actuado sobre el medio rural considerándolo como una zona piloto para la experimentación y la aplicación de iniciativas postproductivistas alejadas de las funciones tradicionales del ámbito rural. Esta política se desarrollaba a partir de tres grandes capitulos.

El Capítulo 1, Estrategias territoriales de desarrollo rural integral, de carácter piloto, basadas en el enfoque ascendente y en la cooperación horizontal, establecía que las estrategias que propusiesen los grupos de acción dentro de este capítulo debían ser integradas y aplicadas mediante un enfoque global, concertado y que buscase la interacción de los agentes territoriales. Además, debían ser coherentes y tener una fundamentación territorial, sobre todo desde el punto de vista económico. Asimismo, debían demostrar su carácter piloto, la posibilidad de transferencia de los métodos de trabajo propuestos y la complementación con otras intervenciones desarrolladas por los programas de desarrollo.

El Capítulo 2, Apoyo a la cooperación interterritorialy transnacional, tenía como finalidad fomentar y apoyar la cooperación entre los territorios con el fin de lograr una masa crítica que hiciese viable un proyecto en común y de buscar complementariedades entre los diferentes territorios. Para ello, se financiaron las acciones de cooperación que supusieron una mejora en la puesta en común de conocimientos técnicos, humanos y financieros de los proyectos de desarrollo. Esta cooperación entre los territorios podía ser interterritorial, es decir, entre varios grupos de acción de un mismo estado, o bien transnacional, o sea, entre varios grupos de acción de diferentes estados miembros.

El Capítulo 3, Integración en una red de todos los territorios rurales de la Comunidad, tengan o no acceso a LEADER + , así como de todos los 
agentes del desarrollo rural, tenía como objeto favorecer la integración de todos los GAL en una red que permitiese el intercambio de logros, experiencias y conocimientos técnicos con el objeto de facilitar el trabajo y desarrollar nuevas iniciativas por parte de los grupos de acción. La Comunicación de la Comisión Europea (2000) indicaba como elementos clave en este proceso la constitución del Observatorio de los territorios rurales, cuyos objetivos eran, entre otros, la recopilación, elaboración y difusión de información y buenas prácticas, la realización de encuentros a escala comunitaria, la elaboración de informes sobre el programa LEADER + y el análisis de los resultados de la aplicación del mismo.

\subsection{La financiación, los ejes de aplicación y las medidas subvencionables en LEADER +}

La financiación de las acciones e iniciativas desarrolladas por los diferentes GAL de Europa, se realizó mediante los fondos asignados a LEADER +. Estas partidas presupuestarias estaban compuestas por las aportaciones de la Unión Europea, las administraciones generales de cada Estado, los gobiernos regionales, las entidades locales y la iniciativa privada.

Según los datos de la Red Española de Desarrollo Rural (2008), durante el periodo de vigencia de LEADER +, la Unión Europea financió a través del Fondo Europeo de Orientación y Garantía Agraria (FEOGA) en la Sección Orientación un total de 2.020,00 millones de euros, a los que habría que sumar las aportaciones realizadas por el resto de los organismos que subvencionan la iniciativa LEADER + .

El proceso de inversión de los fondos económicos seguía tres etapas. En un primer momento, Europa establecía las ayudas económicas que aportaba a sus fondos europeos para el desarrollo rural y, posteriormente, las comunidades autónomas, o demás administraciones regionales responsables de la gestión de estas ayudas, recibían las aportaciones económicas que estaban dirigidas a cada grupo de acción. A continuación, sus agentes gestionaban los fondos, orientando y asesorando a los beneficiarios, que presentaban proyectos innovadores que permitirían desarrollar los ámbitos sociales, culturales y económicos de los 
municipios asociados en cada grupo de acción. Además, cada grupo desarrollaba y aplicaba las acciones dentro de sus prioridades.

Las iniciativas subvencionables por los fondos LEADER + se agrupaban en cuatro ejes, de los que el tercero sólo se desarrollaba en el Programa Nacional LEADER + en los Estados miembro.

Las líneas subvencionables por los fondos LEADER + estaban relacionadas con las áreas de trabajo diseñadas para los grupos de acción. Así pues, el Eje 1 estaba destinado a sufragar las iniciativas llevadas a cabo por los emprendedores de nuevas iniciativas y por las acciones desarrolladas por los grupos de acción en cada territorio. Las acciones de apoyo a la agricultura se integraban dentro de la medida 105 , junto a otras tan dispares como el turismo rural, los servicios a la población y la formación. Por su parte, el Eje 2 se destinaba a financiar los gastos derivados de los programas de cooperación interterritorial y transnacional que desarrollaban varios grupos de acción.

Tabla 1. Ejes de aplicación y medidas subvencionales del programa LEADER +

\begin{tabular}{|l|l|}
\hline Ejes de aplicación & Medidas subvencionales \\
\hline & 101. Adquisición de competencias \\
& 102. Gastos de gestión, funcionamiento administrativo y asistencia \\
& técnica \\
& 103. Servicios a la población \\
Eje 1. Estrategias de desarrollo & 105. Patrimonio \\
& 106. PYlorización de los productos locales y agrarios servicios \\
& 107. Valorización del patrimonio natural y arquitectónico \\
& 108. Turismo \\
& 109. Otras inversiones \\
& 110. Formación y empleo \\
\hline Eje 2. Cooperación & Cooperación interterritorial \\
\hline Eje 3. Puesta en red de los GAL & Cooperación transnacional \\
\hline Eje 4. Gestión, seguimiento y & \\
\hline evaluación & \\
\hline
\end{tabular}

Fuente: Ministerio de Agricultura, Pesca y Alimentación (2008). Disponible en www.mapa.es

Por otro lado, el Eje 3 se fijó para financiar el trabajo en red de los grupos de acción y de otros agentes relacionados con el proceso de 
desarrollo local con los que trabajaban conjuntamente y el Eje 4 estaba destinado a mantener los gastos derivados de los programas de gestión, seguimiento y evaluación de las iniciativas subvencionadas con los fondos LEADER +.

\subsection{El presupuesto disponible de LEADER + en la Unión Europea y en España}

Como se ha indicado arriba, según los datos de la Red Española de Desarrollo Rural (2008), la aportación de la Unión Europea al programa LEADER + se situó en 2.020,00 millones de euros, cantidad que se consignó al Fondo Europeo de Orientación y Garantía Agraria (FEOGA) en la Sección Orientación, es decir, una de las líneas de financiación establecidas por la PAC en 1962. Esta dotación económica se destinó a financiar las acciones puestas en marcha por los 951 grupos de acción europeos durante el periodo de aplicación del programa LEADER + , a la que debería de sumarse las aportaciones realizadas por las administraciones generales de cada Estado miembro, los gobiernos regionales, las entidades locales y la iniciativa privada. La Unión Europea estaba integrada por 15 Estados miembro durante la aplicación de la iniciativa LEADER +. El Estado con mayor número de grupos de acción fue Alemania (154 GAL), seguido de España (145 GAL), Francia (140 GAL) e Italia (135 GAL).

España es quizá el Estado miembro que mostró un mayor carácter rural durante la aplicación de LEADER +, por ello fue el que mayor asignación económica recibió del programa. La cifra se situó en 467,00 millones de euros, lo que representaba el $24,60 \%$ del total de la aportación europea. A continuación le seguían en importancia Italia (13,22\%), Francia $(12,47 \%)$ y Alemania $(12,22 \%)$, mientras que los países que menos aportación recibieron fueron Luxemburgo $(0,10 \%)$, Bélgica $(0,74 \%)$, Dinamarca $(0,80 \%)$ y Suecia $(1,88 \%)$. 
Tabla 2. Aportación de la Unión Europea y Grupos de Acción según el estado. Europa, 2000-2006 (miles de euros, GAL)

\begin{tabular}{|l|c|c|}
\hline \multicolumn{1}{|c|}{ Estado } & Aportación UE & Grupos de acción GAL \\
\hline Alemania & 247.000 & 154 \\
\hline Austria & 71.000 & 56 \\
\hline Bélgica & 15.000 & 12 \\
\hline Dinamarca & 16.000 & 145 \\
\hline España & 467.000 & 58 \\
\hline Finlandia & 52.000 & 140 \\
\hline Francia & 252.000 & 40 \\
\hline Grecia & 172.000 & 28 \\
\hline Holanda & 78.000 & 38 \\
\hline Irlanda & 45.000 & 135 \\
\hline Italia & 267.000 & 4 \\
\hline Luxemburgo & 2.000 & 52 \\
\hline Portugal & 152.000 & 57 \\
\hline Reino Unido & 106.000 & 12 \\
\hline Suecia & 38.000 & 0 \\
\hline Redes de trabajo & 40.000 & 951 \\
\hline Total & 2.020 .000 & $1 / 1$ \\
\hline
\end{tabular}

Fuente: Red Española de Desarrollo Rural (2008). Disponible en http://redrural.es

La iniciativa LEADER + en España se estructuró en un Programa Nacional, cuyo objeto era coordinar a los GAL que participaban en diversas acciones de cooperación interterritorial y en 17 Programas Regionales, cuya finalidad era ejecutar las iniciativas planteadas por cada grupo de acción. En este sentido, en España existían 145 GAL, de los que 140 se incluían en los programas regionales y cinco en la categoría de interterritoriales.

Según los datos del Ministerio de Agricultura, Pesca y Alimentación (2008), los fondos europeos con cargo a la sección Orientación del FEOGA representaron el 62,38\% del total de la inversión, mientras que los públicos de ámbito estatal, autonómico y local supusieron el 37,62\% restante. La financiación total ascendió a 796,52 millones de euros, de los que 496,89 millones de euros fueron financiados por la Unión Europea 
(2) y 299,63 millones de euros por las administraciones generales de cada Estado miembro, los gobiernos regionales, las entidades locales y la iniciativa privada.

Tabla 3. Presupuesto total de LEADER + según las líneas de financiación. España, 20002006 (miles de euros)

\begin{tabular}{|l|c|c|c|}
\hline \multicolumn{1}{|c|}{ Linea } & Total & Europa & España \\
\hline Eje 1. Estrategia de desarrollo & 683.740 & 422.660 & 261.080 \\
\hline Eje 2. Cooperación & 100.250 & 67.330 & 32.920 \\
\hline Eje 3. Puesta en red & 7.500 & 3.600 & 3.900 \\
\hline Eje 4. Gestión, seguimiento y evaluación & 5.030 & 3.300 & 1.730 \\
\hline Total & 796.520 & 496.890 & 299.630 \\
\hline
\end{tabular}

Fuente: Ministerio de Agricultura, Pesca y Alimentación (2008). Disponible en www.mapa.es

Como se puede observar en el cuadro 3, la partida con mayor presupuesto fue el Eje 1 Estrategia de desarrollo con 683,74 millones de euros, es decir, el 85,84\% del total. A éste le siguió en importancia el Eje 2 Cooperación con 100,25 millones de euros, o sea el 12,59\% de la inversión total, mientras que los Eje 3 y 4 representaron únicamente una inversión de 12,53 millones de euros, o lo que es lo mismo, el 1,57\%.

\subsection{La aplicación interna de la iniciativa $L E A D E R+$ en España. El caso de la Comunidad Valenciana y del GAL CEDER- Aitana}

Respecto a la distribución de los fondos y la aplicación del programa LEADER + en el medio rural español se debe indicar que España se estructura internamente en 17 comunidades autónomas que cuentan con un gobierno y una administración propia a partir de la descentralización de las funciones de gestión desde el gobierno del Estado a la comunidades autónomas. En este sentido, las comunidades autónomas con mayor número de grupos de acción adheridos al programa LEADER + durante el periodo 2000-2006 fueron Andalucía (22 GAL), Castilla y León (17 GAL), Galicia (16 GAL) y Castilla-La Mancha (16 GAL), mientras que las regiones con menos grupos de acción fueron el País Vasco (1 GAL), 
La Rioja (2 GAL), Cantabria (2 GAL) y Madrid (3 GAL). Asimismo, estos espacios son los que más y menos subvenciones recibieron del fondo LEADER +, respectivamente.

Según los datos del Ministerio de Agricultura, Pesca y Alimentación (2008), la comunidad autónoma española con mayor asignación presupuestaria para la aplicación del programa LEADER + fue Andalucía con 129,71 millones de euros, a la que siguieron Castilla y León con 100,48 millones de euros, Castilla La Mancha con 82,18 millones de euros y Galicia con 81,75 millones de euros, es decir, las áreas geográficas de mayor carácter rural. Se trata de espacios que se ven fuertemente afectados por la gran dicotomía existente en el territorio español y su cristalización territorial entre las zonas costeras y litorales mediterráneas donde se encuentran las principales ciudades y áreas metropolitanas, entre ellas Barcelona y Valencia, el centro neurálgico funcional y administrativo de Madrid y la existencia de un amplio anillo interior cuyo peso social, administrativo y económico es mucho menor, entre las que destacan las comunidades de Castilla y León y Castilla La Mancha, principalmente.

Si se toma como referencia la autonomía de la Comunidad Valenciana se debe indicar que se trata de un espacio geográfico en el que la iniciativa LEADER + se organizó en torno a ocho grupos de acción como fueron Aitana, Palencia-Mijares, Els Ports/Maestrat, Serrania del Turia, Macizo del Caroig, Rincón de Ademuz, Tierras de Interior y Valle de Ayora-Cofrentes. Según los datos del Ministerio de Agricultura, Pesca y Alimentación (2008), los grupos de acción valencianos recibieron un total de 45,00 millones de euros durante el periodo 2001-2006, de los que el $66,66 \%$ provinieron de los fondos europeos y el $33,34 \%$ restante de los fondos estatales, autonómicos y locales. Los 45,00 millones de euros del programa LEADER + en el espacio valenciano se distribuyeron entre los siguientes ejes: 
Tabla 4. Presupuesto total de LEADER + según la comunidad autónoma. España, 2000-2006 (miles de euros)

\begin{tabular}{|l|c|c|c|}
\hline \multicolumn{1}{|c|}{ Comunidad Autónoma } & Total & Unión Europea & España \\
\hline Andalucía & 129.713 & 86.500 & 43.213 \\
\hline Aragón & 76.000 & 38.000 & 38.000 \\
\hline Asturias & 25.640 & 17.090 & 8.550 \\
\hline Baleares & 9.200 & 4.600 & 4.600 \\
\hline Canarias & 22.948 & 15.300 & 7.648 \\
\hline Cantabria & 13.428 & 9.000 & 4.428 \\
\hline Castilla La Mancha & 82.188 & 54.000 & 28.188 \\
\hline Castilla y León & 100.489 & 69.000 & 31.489 \\
\hline Cataluña & 50.000 & 25.000 & 25.000 \\
\hline Extremadura & 48.000 & 32.000 & 16.000 \\
\hline Galicia & 81.750 & 54.500 & 27.250 \\
\hline Madrid & 12.600 & 6.300 & 6.300 \\
\hline Murcia & 17.850 & 11.900 & 5.950 \\
\hline Navarra & 17.000 & 8.500 & 8.500 \\
\hline La Rioja & 11.200 & 5.600 & 5.600 \\
\hline Comunidad Valenciana & 45.000 & 30.000 & 15.000 \\
\hline País Vasco & 11.920 & 6.000 & 5.920 \\
\hline Programa Nacional & 41.594 & 23.600 & 17.994 \\
\hline Total & 796.520 & 496.890 & 299.630 \\
\hline
\end{tabular}

Fuente: Ministerio de Agricultura, Pesca y Alimentación (2008). Disponible en www.mapa.es

1. Eje 1. Estrategias de Desarrollo, con 40,76 millones de euros.

2. Eje 2. Cooperación, con 4,00 millones de euros.

3. Eje 4. Gestión, Seguimiento y Evaluación, con 0,24 millones de euros.

Entre las principales deficiencias en la aplicación de la iniciativa LEADER + en las autonomías españolas se debe destacar que las acciones subvencionadas y puestas en marcha en la mayor parte de los grupos de acción no se han desarrollado bajo ningún proyecto estratégico territorial. Por ello, las acciones implementadas han mostrado un elevado grado de desconexión entre sí y han estado poco justificadas desde 
un punto de vista metodológico y estratégico. Además, al no incidir directamente sobre la cuestión agraria en la mayoría de los casos, se han visto deslegitimadas ante la población rural que las ha considerado como acciones implementadas desde una entidad lejana que no había recabado sus consideraciones a través de procesos de difusión, debate y participación ciudadana.

El GAL CEDER-Aitana agrupó durante el desarrollo de LEADER + a 55 municipios de la Comunidad Valenciana. Su territorio, con una extensión de $1.153 \mathrm{~km}^{2}$, se articula en dos áreas de desarrollo como son el eje industrial interior y el eje turístico litoral. En esta área destacan los municipios de escaso peso demográfico. De ellos, 41 municipios tienen menos de 750 habitantes, seis tienen entre 751 y 1.000 habitantes, tres municipios tienen entre 1.001 y 2.000 habitantes, dos tienen entre 2.001 y 5.000 habitantes y tan sólo tres municipios tienen más de 5.000 habitantes.

Según los datos del GAL CEDER-Aitana (2008), la aplicación del programa LEADER + en sus municipios supuso una inversión de 16.036 miles de euros, de los es el $58,25 \%$ fueron fondos privados y el $41,72 \%$ por fondos públicos. La mayor parte de esta inversión se realizó dentro del Eje 1 Estrategias de desarrollo, en el que destacó la inversión realizada en las medidas de Valorización de productos agrarios, Turismo rural, Servicios a la población y Patrimonio natural.

Maps 1

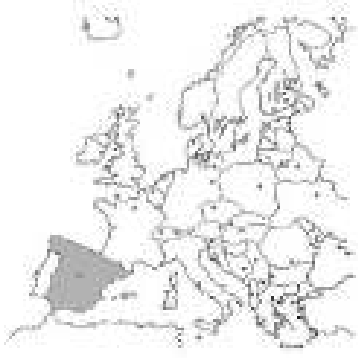

Españ
Mapa 2

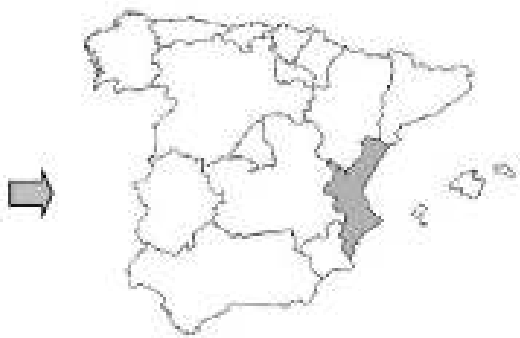

Comunidad Valenciana

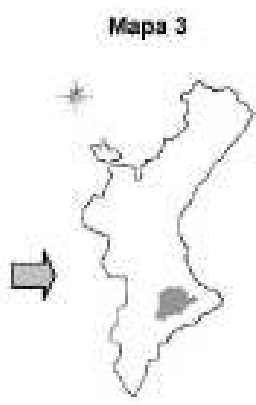

CEDER-Aitana

Figura 1. Marco espacial de referencia

Fuente: Elaboración propia. 
Como muestra se debe indicar que dentro de la medida de Valorización de productos agrarios se subvencionó la modernización de las instalaciones de las cooperativas agrarias y de las empresas agroalimentarias, la compra de maquinaria, la realización de ferias agrarias y la implantación de las nuevas tecnologías.

Tabla 5. Presupuesto total de LEADER + según los ejes y medidas.

GAL CEDER-Aitana, 2000-2006 (miles de euros)

\begin{tabular}{|l|c|}
\hline \multicolumn{1}{|c|}{ Ejes y medidas subvencionadas } & $\begin{array}{c}\text { Inversión total } \\
\text { (Miles de } € \text { ) }\end{array}$ \\
\hline Eje 1. Estrategias de desarrollo & 14.777 \\
\hline 103. Servicios a la población & 2.846 \\
\hline 104. Patrimonio & 931 \\
\hline 105. Valorización de productos locales y agrarios & 4.913 \\
\hline 106. Pequeñas y medianas empresas y artesanía & 1.012 \\
\hline 107. Valorización patrimonio natural y arquitectónico & 1.168 \\
\hline 108. Turismo & 3.452 \\
\hline 109. Otras inversiones & 118 \\
\hline 110. Formación y empleo & 337 \\
\hline Eje 2. Cooperación & 414 \\
\hline Cooperación interterritorial & 414 \\
\hline Eje 4. Gestión, seguimiento y evaluación & 845 \\
\hline Apoyo técnico y gastos de estructura técnica & 845 \\
\hline Total & 16.036 \\
\hline
\end{tabular}

Fuente: GAL CEDER-Aitana (2008).

Dentro de la medida de Turismo se subvencionó la puesta en marcha de alojamientos de turismo rural y de establecimientos de restauración, la edición de material para la promoción turística, la dotación de colecciones museográficas y la puesta en marcha de oferta complementaria de ocio y tiempo libre a través de empresas especializadas en la materia. A estas actuaciones se deben sumar las realizadas en otras medidas con el objeto de recuperar y valorizar el patrimonio natural, cultural y arquitectónico como son los castillos con fines turísticos. 
Además, dentro de la medida de Servicios a la población se apoyó la puesta en marcha de centros de atención geriátrica como las residencias y los centros de día, los centros de consulta médica y de atención a pacientes de alzheimer y el acondicionamiento de centros sociales y de viviendas municipales.

Como se ha indicado arriba, las inversiones en materia agraria realizadas a través de la iniciativa LEADER han ocupado un lugar secundario entre 1991 y 2006. La inversión total, pública y privada, realizada durante este periodo se ha situado en 41,07 millones de euros. Si se analiza la inversión realizada a través de los ejes de Turismo rural y Valorización de productos agrarios se puede observar que el primero ha aglutinado el $39,57 \%$, mientras que el segundo ha adherido al $30,73 \%$ del total.

Tabla 6. Comparativa según ejes. GAL CEDER-Aitana, 1991-2006 (miles de euros)

\begin{tabular}{|l|c|c|c|c|}
\hline & LEADER I & LEADER II & LEADER + & Total 1991-2006 \\
\hline Eje Turismo rural & 9.133 & 3.669 & 3.452 & 16.254 \\
\hline Eje Valorización de productos agrarios & 330 & 2.797 & 4.913 & 12.623 \\
\hline Total fase & 10.853 & 14.188 & 16.036 & 41.077 \\
\hline
\end{tabular}

Fuente: GAL CEDER-Aitana (2008).

La mayor diferencia se registró durante la aplicación de LEADER I, ya que el eje de Turismo rural supuso un porcentaje total del $84,15 \%$ respecto al total de inversión realizada, mientras que el eje Valorización de productos agrarios tan solo significó el 3,04\%.

Ante la falta de una mayor implicación del programa LEADER en el desarrollo de la actividad agraria y la superación de los condicionantes negativos que afectan actualmente a la agricultura, un grupo de entidades del área de acción del GAL CEDER-Aitana han puesto en marcha una iniciativa para la recuperación y puesta en valor de las parcelas agrarias que durante los últimos años habían dejado de cultivarse a causa del descenso de actividad y de la avanzada edad de los agricultores. Se trata del proyecto Microviña. Esta iniciativa ha sido implementada por la Asociación Elviart y la bodega Celler La Muntanya SL del municipio de Muro y ha conseguido convertir estas debilidades en nuevas oportunidades para los agricultores del medio rural. 
El proyecto Microviña consiste en la implantación del cultivo de la vid en las tierras agrarias en desuso cuyo norte es la obtención de producciones de elevada calidad tras la realización de diversos controles. La iniciativa se desarrolla a partir de la implicación de los agricultores minifundistas y de los vecinos que tienen pequeños terrenos sin producir para que introduzcan las viñas que permitan la obtención de uva para su ulterior transformación en vinos tinto y blanco por parte de la empresa Celler de La Muntanya SL bajo las marcas de Almoroig y Albir. Además, fruto del proyecto Microviña, se ha elaborado el producto D'Olives, un aceite de oliva virgen obtenido a partir de las variedades autóctonas cultivadas en pequeñas parcelas agrarias de varios municipios rurales. Se trata de un producto del que se han distribuido 4.000 botellas de medio litro de aceite durante el año 2008 en restaurantes nacionales e internacionales.

Esta iniciativa ha permitido demostrar que la actividad agraria minifundista puede convertirse en un sistema de explotación rentable siempre y cuando se aplique bajo criterios de al ta calidad y profesionalidad técnica, agraria y empresarial y que sirve, además, para sostener el paisaje rural y proteger el ambiente. Se trata de una iniciativa que se basa en el mercado, no en la subvención.

\subsection{La iniciativa LEADER en el horizonte 2007-2013}

La iniciativa LEADER ha tomado un nuevo impulso tras el cierre del programa LEADER +. Actualmente la Unión Europea está integrada por 25 Estados miembro, lo que ha supuesto un cambio importante en la distribución de los fondos y las asignaciones económicas de las políticas europeas. La nueva política europea de desarrollo rural está regulada por el Reglamento CE 1698/2005 en el que se define el Fondo Europeo Agrícola de Desarrollo Rural (FEADER). Este reglamento establece tres ejes de actuación prioritarios, así como un eje transversal. Son los siguientes:

- Eje 1. Aumento de la competitividad del sector agrícola y forestal.

- Eje 2. Gestión de la tierra (protección del ambiente). 
- Eje 3. Diversificación de la economía rural y calidad de vida en las zonas rurales.

- Eje transversal. Iniciativa LEADER.

El Reglamento CE 1698/2005 marca unos porcentajes mínimos de gasto en cada eje respecto a la asignación económica total de FEADER para cada Estado miembro. El porcentaje establecido para la agricultura es el más bajo de los ejes prioritarios y debe de compartirse con la mejora forestal. Éste se sitúa en el $10 \%$, mientras que el porcentaje para la protección del ambiente es del $25 \%$. Con ello, queda de manifiesto la continuidad de la política de desarrollo rural de la Unión Europea iniciada con la reforma de la PAC y en la que la agricultura ocupa un papel secundario.

Los acuerdos preeliminares firmados a principios de 2005 indicaban que el porcentaje mínimo establecido para el Eje 1 era del 15\%. La revista Actualidad LEADER publicó en su número 28, correspondiente a junio de 2005, varios artículos firmados por las organizaciones Asociación Agraria de Jóvenes Agricultores (ASAJA), Coordinadora de Organizaciones de Agricultores y Ganaderos (COAG), Unión de Pequeños Agricultores y Ganaderos (UPA) y Confederación de Cooperativas Agrarias de España (CCAE) en los que se analizaban los acuerdos preeliminares firmados a principios de 2005. Entre sus propuestas para este nuevo horizonte de la política de desarrollo rural europea destacaban la necesidad de reconocer al sector agrario como motor de desarrollo económico rural, de incrementar la participación de los agricultores en la toma de decisiones, de apoyar el desarrollo de las explotaciones familiares y de incrementar el porcentaje mínimo de gasto del Eje 1.Como se puede observar, las demandan de los agricultores no han sido atendidas, ya que este porcentaje mínimo se ha reducido sustancialmente, pasando del $15 \%$ al $10 \%$ final

Este nuevo periodo de aplicación se desarrollará entre 2007 y 2013 y ha supuesto una serie de cambios respecto a las convocatorias anteriores. De entrada, la aplicación de la iniciativa LEADER ha supuesto una reducción de los municipios incluidos en cada GAL, su reestructuración 
y un cambio importante en las líneas de financiación. Se han establecido las siguientes: Creación y desarrollo de microempresas, Fomento de las actividades turísticas, Prestación de servicios básicos para la economía y la población rural, Renovación y desarrollo de pueblos y Conservación y mejora del patrimonio rural.

Durante este nuevo periodo de aplicación, los gobiernos de las distintas regiones europeas tendrán que realizar un mayor esfuerzo económico, ya que la aportación de la Unión Europea se reducirá durante los próximos años. En este sentido, si se considera la aplicación de la iniciativa LEADER en la Comunidad Valenciana (España) las aportaciones se determinan de la siguiente forma.

- Unión Europea. Fondo Europeo de Agricultura y Desarrollo Rural (FEADER): $36,50 \%$ de los fondos.

- Gobierno de España. Ministerio de Agricultura, Pesca y Alimentación: $1,00 \%$ de los fondos.

- Gobierno valenciano. Fondo Estrategias para los Territorios Rurales Valencianos (RURALTER): $62,50 \%$ de los fondos.

Ante la reducción de los municipios incluidos en las áreas de aplicación de LEADER, los gobiernos autonómicos españoles han tenido que poner en marcha una serie de iniciativas paralelas con las que apoyar su desarrollo social y económico. En concreto, el gobierno de la Comunidad Valenciana ha puesto en marcha los fondos RURALTERPaisaje y RURALTER-Dinamización que se sustentan en la aplicación del Programa de Desarrollo Rural de la Comunidad Valenciana (2007-2013) y cuya líneas de apoyo son las mismas que se establecen en LEADER.

\section{Conclusiones}

El programa Liasions Entre Activités de Development de l'Economie Rural (LEADER) es una iniciativa desarrollada por la Unión Europea y los Estados miembro que se puso en marcha en el año 1991 con el objeto de promover el desarrollo de los espacios rurales europeos. Para 
ello, se han implementado de forma completa tres fases de aplicación de la iniciativa que se corresponden con los programas LEADER I (1991-1993), LEADER II (1994-1999) y LEADER + (2000-2006), así como la actual renovación de LEADER y su aplicación en el horizonte 2007-2013.

Los programas LEADER, especialmente LEADER +, se han centrado en apoyar la puesta en marcha de medidas para la mejora de los servicios a la población, la valorización de los productos locales y agrarios, el turismo rural, la valorización del patrimonio natural y arquitectónico y la formación y el empleo. En síntesis, como se ha podido observar, se trata de programas que han promovido un nuevo modelo de desarrollo rural sustentado en la multifuncionalidad del medio rural y no en la multifuncionalidad de la actividad agraria.

Es necesario preservar el medio rural europeo a partir del desarrollo de un modelo rural ético, comprometido y sustentado en la agricultura con la finalidad de potenciar el crecimiento planificado y ordenado de este tipo de áreas. Este modelo de desarrollo permitirá hacer frente al deterioro del ambiente en las áreas rurales como consecuencia de los impactos negativos generados por el afianzamiento y explotación del ocio, el tiempo libre y el turismo rural, así como por el desarrollo de las urbanizaciones y segundas residencias para la población urbana. Además, permitirá preservar el paisaje y el patrimonio tradicional rural, así como las especies vegetales y animales.

La aplicación de las políticas homogeneizadas de la Unión Europea deben enmarcarse en la concreción y puesta en marcha de planes de desarrollo estratégicos que adecuen las políticas globales europeas a las cuestiones y características locales a través de acciones que muestren sinergias entre sí y que permitan el desarrollo de las áreas rurales europeas desde la escala básica del Grupo de Acción Local, incidiendo de una forma especial en la agricultura.

\section{Notas}

(1) Este artículo se enmarca en la tesis doctoral 'Caracterización socioeconómica, políticas y proyectos para el desarrollo de la comarca funcional de l'Alcoià, el Comtat y la Foia de Castalla. Una apuesta por 
la planificación estratégica territorial' que ha sido realizada gracias a la concesión de una Ayuda a la Investigación (Convocatoria 2007) por parte del Instituto Alicantino de Cultura Juan Gil-Albert (Excma. Diputación de Alicante).

(2) Como se puede observar, existe una ligera diferencia en la aportación realizada por la Unión Europea en España con cargo al programa LEADER +. Esta diferencia se debe a las distintas particularidades establecidas por las bases de datos de la Red Española de Desarrollo Rural y del Ministerio de Agricultura, Pesca y Alimentación. 


\section{Bibliografía}

Barato, P. 2005. Diversificación para los agricultores. En: Actualidad LEADER 28: 14-15. Comisión Europea. 2003. Dictamen del Comité Económico y Social de la Unión Europea El futuro de las zonas de montaña en la Unión Europea (2003/C61/19).

Conselleria d'Agricultura, Pesca i Alimentació. 1999. Programa Regional de la Iniciativa Comunitaria Leader + en la Comunidad Valenciana (2000-2006). Valencia: Generalitat Valenciana: 116. Disponible en http://redrural.mapa.es

Crecente, R. 2002. Ordenación del espacio rural como instrumento de la multifuncionalidad. En: Jornada técnica Libro Blanco de la agricultura y el desarrollo rural. Santiago de Compostela, 10 de julio de 2002.

Farinós, J. 2004. Los territorios rurales en el cambio de siglo. En: Geografia humana. Barcelona, Ariel: 332-393.

García, A. 2007. Precios en origen y precios en destino. En: Supermercados, no gracias. Barcelona, Icaria: 65-69.

Gómez, J. D. 2008. Cooperativas agrarias y desarrollo rural. En: Master de desarrollo local e innovación territorial. Alicante, Universidad de Alicante.

López, M. 2005. Impulsar el asociacionismo agrario. En: Actualidad LEADER 28: 16-17.

Martín, R. 2005. Hacia un cooperativismo polivalente. En: Actualidad LEADER 28: 20-21

Ministerio de Agricultura, Pesca y Alimentación. 2008. La aplicación de los programas europeos en España. Recurso electrónico disponible en www.mapa.es

Pliego, J. 2002. El desarrollo rural como motor de futuro del ámbito agrícola. En: Visión del futuro de la agricultura europea. Madrid, MAPA: 155-168.

Ramos, L. 2005. Los agricultores, protagonistas. En: Actualidad LEADER 28: 18-19

Red Española de Desarrollo Rural REDR. 2008. La aplicación de los programas europeos en España. Recurso electrónico disponible en http://redrural.es

Romero, J. J. 2002. Los efectos de la politica agraria europea. Un análisis critico. Bilbao, Desclée de Brouwer: 326.

Segrelles, J. A. 2007. La calidad agroalimentaria de la Unión Europea (UE): un instrumento proteccionista contra la competencia comercial de la agricultura latinoamericana. En: $X I$ Encuentro de Geógrafos de América Latina-EGAL, Bogotá, 26-30 de marzo de 2007.

Segrelles, J. A. 2008. Viejos y nuevos enfoques del desarrollo rural. En: Master de desarrollo local e innovación territorial. Alicante, Universidad de Alicante.

Recibido. abril 2008

Aceptado: septiembre 10 de 2008 